\title{
Quantification des risques de contamination et d'exposition externe du personnel en radiothérapie interne vectorisée par iode-131
}

\author{
S. Guillot ${ }^{1 \star}$, P. Tylski ${ }^{1}$, C. Scheiber ${ }^{2}$, C. Bournaud-Salinas ${ }^{2}$, C. Bolot ${ }^{3}$, C. Harthe ${ }^{4}$ et P. Jalade ${ }^{1}$ \\ 1 Hospices Civils de Lyon, Service de Physique Médicale et Radioprotection, Groupement Hospitalier Sud, 165 Chemin du Grand Revoyet, \\ 69495 Pierre-Bénite Cedex, France. \\ 2 Hospices Civils de Lyon, Groupement Hospitalier Est, Centre de Médecine Nucléaire, 59 boulevard Pinel, 69677 Bron Cedex, France. \\ 3 Hospices Civils de Lyon, Groupement Hospitalier Est, Radiopharmacie, 59 boulevard Pinel, 69677 Bron Cedex, France. \\ 4 Hospices Civils de Lyon, Groupement Hospitalier Est, Centre de Biologie Est, 59 boulevard Pinel, 69677 Bron Cedex, France.
}

Reçu le 15 septembre 2015 - Accepté le 17 juin 2016

\begin{abstract}
Résumé - Afin de quantifier les sources d'exposition et de contamination induites par les patients traités à l'iode-131 sur le personnel, nous réalisons au cours de l'hospitalisation des mesures de débit de dose et de contamination atmosphérique, surfacique et cutanée sur douze patients recevant une activité de 3,7 GBq, six ayant bénéficié d'une stimulation par TSHr et six par sevrage d'hormone thyroïdienne. Les valeurs maximales sont mesurées à $t=4 \mathrm{~h}$ après la prise d'iode, avec en moyenne, un débit de dose à 1 mètre de $133 \mu \mathrm{Sv} \mathrm{h}^{-1}$, une concentration salivaire de $8,8 \mathrm{MBq} \mathrm{g}^{-1}$, une activité surfacique cutanée de $46 \mathrm{~Bq} \mathrm{~cm}^{-2}$, une concentration atmosphérique de $183 \mathrm{~Bq} \mathrm{~m}^{-3}$ et une contamination des sols inférieure à $0,5 \mathrm{~Bq} \mathrm{~cm}^{-2}$. Les mesures montrent une grande variabilité entre les patients, résultant des différences individuelles physiologiques d'élimination de l'iode-131 et de l'application inégale des consignes d'hygiène. Les contaminations dans l'air de la chambre ont un impact dosimétrique faible par rapport à l'exposition externe provenant du patient mais elles peuvent expliquer les contaminations internes du personnel. Les consignes sont donc de limiter les passages dans la chambre lors des 24 premières heures et faire appliquer de façon stricte les consignes d'hygiène pour tous les patients.
\end{abstract}

\begin{abstract}
Exposure and contamination assessment of nuclear medical staff during radioiodine therapy for thyroid cancer. To assess exposure and contamination induced by patients treated with iodine-131 on nuclear medical staff, we performed several measurements during patient hospitalization: the dose rate, skin contamination, and room air and ground contamination. These measurements were performed for 12 patients treated with $3.7 \mathrm{GBq}$ of iodine131, 6 stimulated by TSHr and 6 by thyroid hormone withdrawal. Maximum values were measured $4 \mathrm{~h}$ after iodine administration, with a mean dose rate of $133 \mu \mathrm{Sv} \mathrm{h}^{-1}$ at 1 meter, a mean activity concentration of $8.8 \mathrm{MBq} \mathrm{g}^{-1}$ in saliva, a mean room air concentration of $183 \mathrm{~Bq} \mathrm{~m}^{-3}$ and a ground concentration below $0.5 \mathrm{~Bq} \mathrm{~cm}^{-2}$. These results highlight a wide inter-patient variability, resulting from individual physiological differences in iodine elimination and from various applications of hygiene instructions. Room air contamination has a small impact in terms of staff exposure, compared with external exposure from the patient. Nevertheless, it is probably the main origin of internal contamination of nuclear medical staff. It is now recommended for the staff to limit visits in the room in the first $24 \mathrm{~h}$, and for all patients to strictly follow hygiene instructions.
\end{abstract}

Keywords: Iodine-131 / contamination / thyroid / therapy

\section{Introduction}

La radiothérapie interne vectorisée (RIV) par iode-131 entraîne une exposition externe, mais peut également induire une contamination du personnel soignant lors des soins donnés pendant l'hospitalisation. C'est le cas dans l'unité de thérapie

\footnotetext{
^ sebastien.guillot@chu-lyon.fr
}

du centre de médecine nucléaire du Groupement Hospitalier Est des Hospices Civils de Lyon, où les examens de radiotoxicologie des urines sont ponctuellement positifs pour une partie du personnel malgré l'utilisation d'équipement de protection (gants, sur-blouse) et l'absence de contaminations mains/pieds en sortie de zone. L'analyse des risques de contamination par des radionucléides pour le personnel du secteur de thérapie 
reposait jusqu'à présent sur des données publiées (Ibis et al., 1992). Ibis et al. trouvaient des activités significatives d'iode131 dans la salive, la transpiration, l'air exhalé par les patients et dans l'air de la chambre.

Afin de quantifier les risques d'exposition et de contamination propres au service, cinq types de mesures ont été réalisés :

- débit de dose à un mètre du patient ;

- contamination atmosphérique avec des prélèvements d'air ;

- contamination dans la salive à l'aide de prélèvements salivaires ;

- contamination cutanée à l'aide de frottis ;

- contamination du sol de la chambre et de la salle de bain par comptage des consommables utilisés pour le nettoyage.

Ces mesures ont été réalisées pour deux situations thérapeutiques différentes en fonction du mode de stimulation thyréotrope choisi : la stimulation endogène, par sevrage en hormones thyroïdiennes, et la stimulation exogène, par injection de TSH humaine recombinante (TSHr). Le choix du mode de préparation à la RIV par iode-131 est guidé par l'étendue de la maladie, et l'état général du patient. Il influence la période biologique de l'iode-131 et la durée d'hospitalisation.

L'objectif de cette étude est de déterminer si le type de stimulation des cellules thyroïdiennes par sevrage ou TSHr nécessite des adaptations de prise en charge du point de vue de la radioprotection, de mieux identifier les vecteurs de contamination et de mettre en place des actions complémentaires de prévention.

\section{Matériel et méthodes}

\subsection{Patients étudiés}

Douze patients volontaires, 4 hommes et 8 femmes âgés de 29 à 70 ans (44 ans en moyenne) ont participé à cette étude. Tous les patients ont reçu, par voie orale, une dose forfaitaire d'iode-131 de 3,7 GBq. Les 12 patients sont séparés en deux groupes : 6 patients hospitalisés 3 jours ayant reçu une stimulation par TSHr et 6 patients hospitalisés 5 jours après sevrage en hormones thyroïdiennes.

\subsection{Mesures réalisées}

Les mesures de débit d'équivalent de dose ambiant, les prélèvements atmosphériques, cutanés et salivaires sont réalisés à 4,24 et $48 \mathrm{~h}$ après l'absorption d'iode-131 pour tous les patients. Pour les patients hospitalisés 5 jours, des mesures supplémentaires à 72 et $96 \mathrm{~h}$ ont été réalisées, sauf pour les prélèvements atmosphériques. Ces mesures ont été effectuées dans 8 chambres différentes mais très similaires en volume, surface (environ $12 \mathrm{~m}^{2}$ ) et agencement (salle de bains avec WC attenant à chaque chambre). L'extraction d'air s'effectue grâce à une seule bouche d'aspiration présente dans chaque salle de bain à un débit moyen de $227 \mathrm{~m}^{3} \mathrm{~h}^{-1}$ entraînant 6 à 7 renouvellements d'air par heure et une dépression par rapport au couloir.
Les prélèvements d'air ou de salive, les frottis ont été mesurés avec un activimètre ou un spectromètre gamma. Le choix de l'appareil de mesure, la méthode de détermination des rendements de comptage et les résultats obtenus figurent en annexe.

\subsubsection{Mesure de débit d'équivalent de dose ambiant}

Les mesures de débit d'équivalent de dose ambiant $\left(\mathrm{H}^{*} 10\right)$ en $\mu \mathrm{Sv} \mathrm{h}^{-1}$ à un mètre du patient sont effectuées avec le patient assis dans le fauteuil ou au bord du lit. Le radiamètre utilisé est un FH40 GL-10 vérifié et étalonné.

\subsubsection{Prélèvements atmosphériques}

La contamination atmosphérique a été quantifiée grâce à des prélèvements d'air sur cartouche à charbon actif modèle TE2B imprégné de triéthylène diamine (TEDA). Le rendement en rétention du filtre est proche des $100 \%$. Deux prélèvements sont effectués : un dans la chambre et l'autre dans la salle de bain afin de vérifier si la contamination s'équilibre d'une pièce à l'autre ou s'il y a une pièce où la contamination stagne. Ces prélèvements ont été réalisés grâce à un préleveur d'air bas débit de type DF-AB-40L (APVL). Chaque prélèvement d'air, effectué à un débit de 30 litres par minute, a duré entre 35 et $60 \mathrm{~min}$. Les mesures de la contamination de l'air sont en $\mathrm{Bq} \mathrm{m}^{-3}$.

\subsubsection{Prélèvements salivaires}

Les prélèvements de salive ont été réalisés grâce à un dispositif dédié de type Salivette ${ }^{\circledR}$ (Sarstedt). Le patient mâche le coton pendant une minute. Une pesée du dispositif de prélèvement à l'aide d'une balance électronique MDE $124 \mathrm{~S}$ (Sartorius) avant et après le prélèvement permet de déduire la masse nette de salive prélevée. La mesure finale de l'activité dans la salive est exprimée en $\mathrm{Bq} \mathrm{g}^{-1}$.

\subsubsection{Prélèvements cutanés}

Les frottis cutanés réalisés à l'aide de cotons « boule» imprégnés avec $1 \mathrm{ml}$ d'alcool à $70 \%$ sont effectués au niveau de la paume de chaque main et du front qui sont les zones de peau où l'activité surfacique semble la plus importante (Ibis et al., 1992). L'activité surfacique la plus élevée entre les deux mains sera prise en compte. Les frottis sont réalisés à l'aide de gabarits pour avoir une surface frottée de $10 \mathrm{~cm}^{2}$. Une fois le prélèvement effectué, le coton est placé dans un flacon cylindrique de $40 \mathrm{~cm}^{3}$ en polypropylène de type « poudrier », la surface du coton en contact avec la peau vers le bas. L'ensemble est placé directement sur le cristal du spectromètre gamma au centre de celui-ci. La mesure finale est exprimée en $\mathrm{Bq} \mathrm{cm}^{-2}$ de peau. 


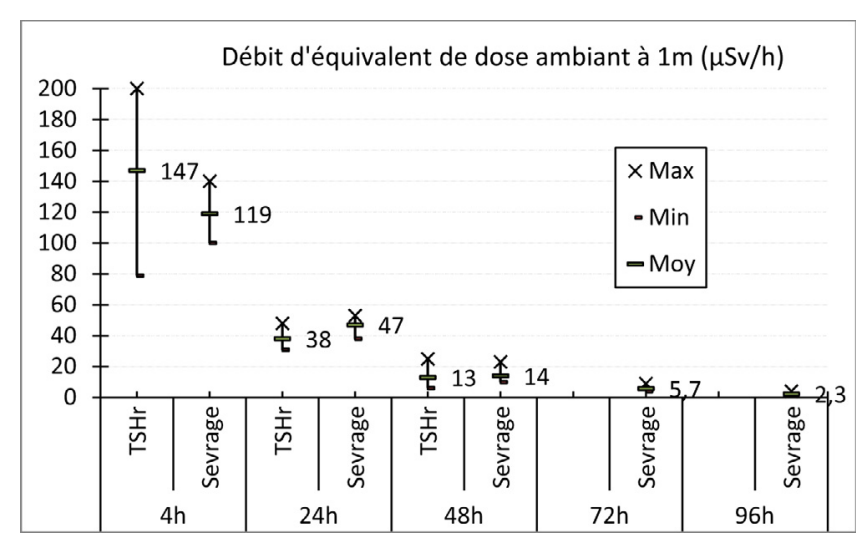

Figure 1. Débit d'équivalent de dose ambiant à 1 mètre. Dose rate at 1 meter.

\subsubsection{Frottis du sol de la chambre}

La contamination du sol de la chambre et de la salle de bains est évaluée en fin de séjour en récupérant et mesurant les gazes pré-imprégnées (utilisées pour le balayage à sec) et les bandeaux de lavage.

Ceux-ci sont placés dans des poudriers en polypropylène et placés directement sur le cristal du spectromètre gamma.

Les activités de la gaze pré-imprégnée et du bandeau utilisés pour le sol de la chambre sont additionnées. On considère une incertitude de $5 \%$ sur la surface frottée pour la salle de bain et de $10 \%$ pour la chambre. Les résultats sont exprimés en $\mathrm{Bq} \mathrm{cm}^{-2}$.

\section{Résultats}

Les résultats des mesures, frottis, prélèvements sont présentés en fonction du temps après l'absorption de la dose thérapeutique et sont divisés en deux groupes : TSHr ou Sevrage. Pour chaque groupe est indiqué la moyenne et les mesures minimale et maximale.

\subsection{Débit d'équivalent de dose à 1 mètre}

Les débits d'équivalent de dose ambiant à 1 mètre du patient reportés en Figure 1 sont compris entre 79 et $200 \mu \mathrm{Sv} \mathrm{h}^{-1}$ avec une moyenne à $133 \mu \mathrm{Sv} \mathrm{h}^{-1}$ à $t=4 \mathrm{~h}$. La dispersion est plus importante pour le groupe TSHr.

La diminution du débit de dose au cours du temps est d'allure mono-exponentielle. Par ajustement avec une fonction exponentielle, il est possible d'estimer les périodes effectives des deux groupes. On obtient 13,2 $\pm 3,5 \mathrm{~h}$ pour le groupe TSHr et $15,5 \pm 2,3 \mathrm{~h}$ pour le groupe Sevrage.

\subsection{Contamination atmosphérique}

Les mesures de contamination de l'air sont présentées en Figures 2 et 3 . Il existe de très grandes variabilités de mesure d'un patient à l'autre, les concentrations pouvant s'échelonner de 31 à $745 \mathrm{~Bq} \mathrm{~m}^{-3}$ à $t=4 \mathrm{~h}$ (Figure 2A). Les concentrations
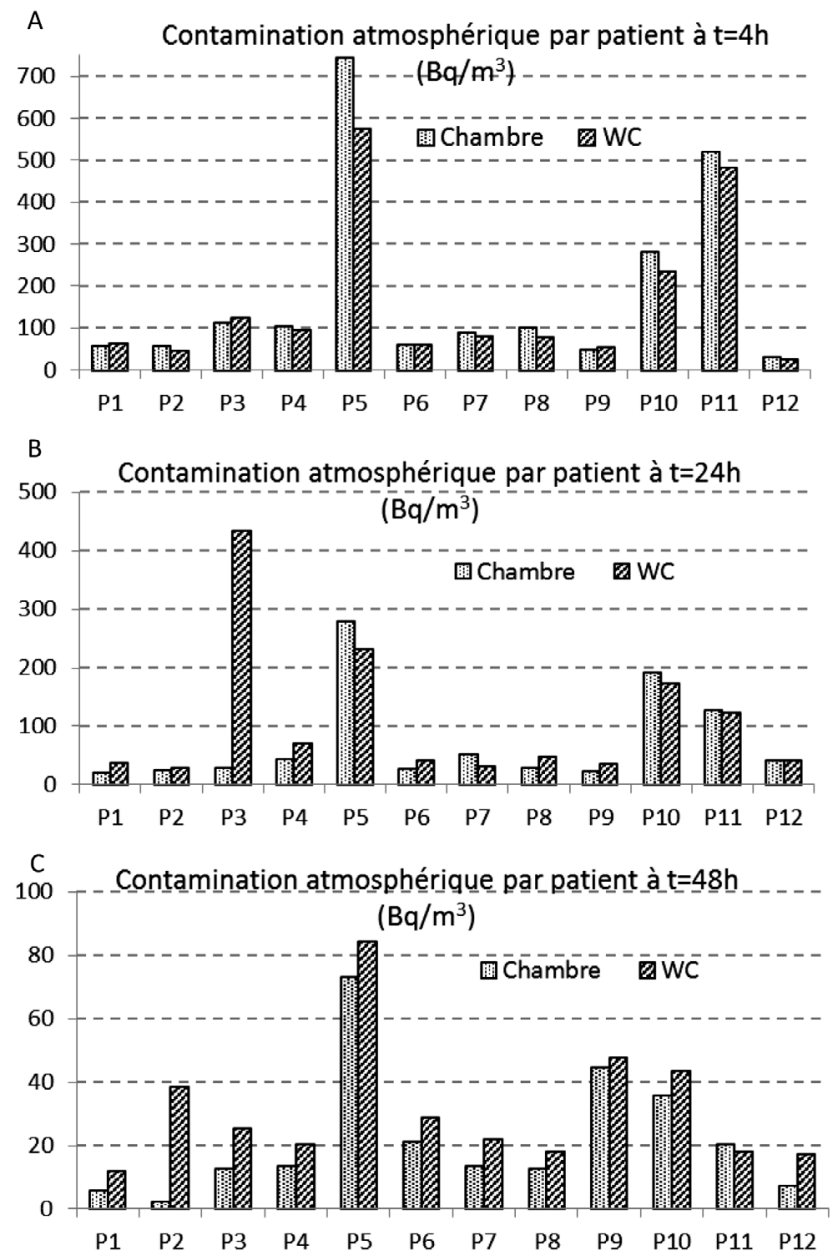

Figure 2. Contamination atmosphérique dans la chambre et les WC à 4,24 et $48 \mathrm{~h}$.

Bedroom and toilet air concentration at 4, 24 and $48 \mathrm{hr}$.

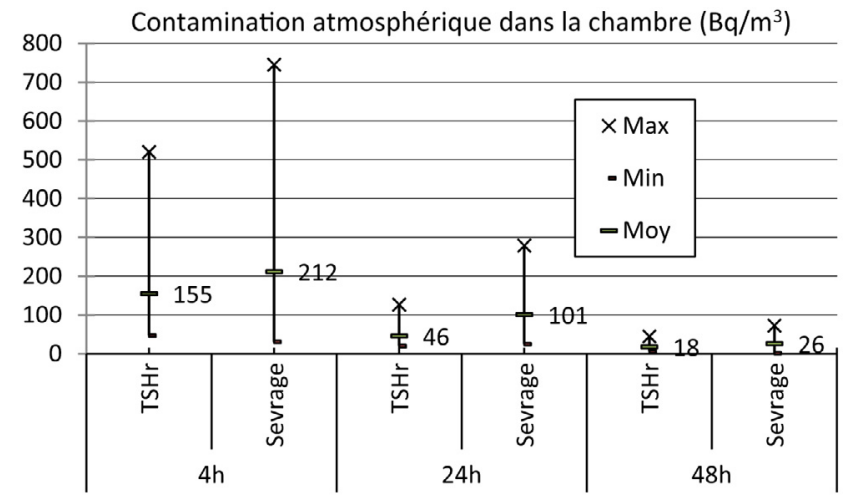

Figure 3. Contamination atmosphérique dans la chambre. Bedroom air concentration.

atmosphériques sont proches dans les chambres et les WC à $t=4 \mathrm{~h}$ et $t=24 \mathrm{~h}$. On constate une concentration dans l'air beaucoup plus élevée dans les WC à $t=24 \mathrm{~h}$ pour le patient No. 3 (Figure 2B). À $t=48 \mathrm{~h}$ la concentration atmosphérique est globalement plus élevée dans les WC (Figure 2C). 


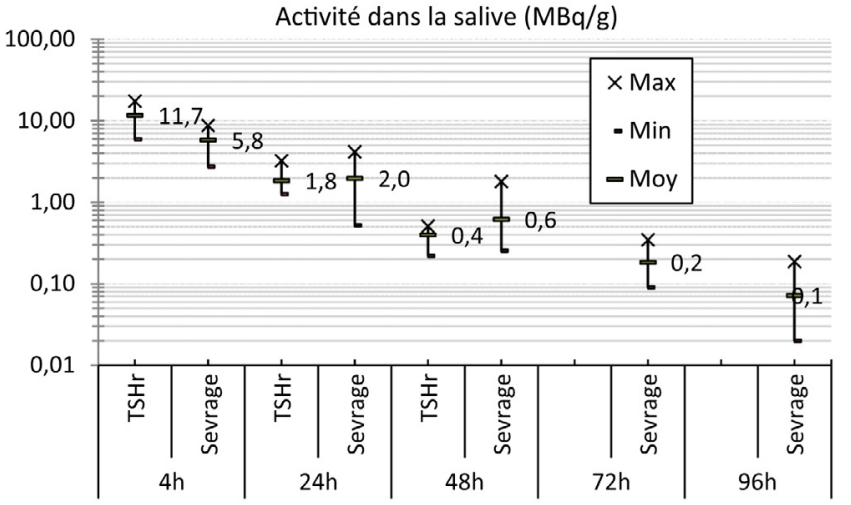

Figure 4. Activité dans la salive.

Saliva activity.

\subsection{Contamination de la salive}

L'activité dans la salive représentée sur la Figure 4 est de l'ordre du MBq par gramme de salive avec une grande variabilité d'un patient à l'autre. Les concentrations sont les plus élevées à $t=4 \mathrm{~h}$ pour le groupe TSHr. L'activité diminue plus rapidement dans le groupe $\mathrm{TSHr}$.

\subsection{Contamination cutanée}

L'activité surfacique au niveau des mains (Figure 5A) à $t=4 \mathrm{~h}$ ne dépasse pas $125 \mathrm{~Bq} \mathrm{~cm}{ }^{-2}$ pour le groupe TSHr. Un patient du groupe sevrage (patient No. 5) est très au-dessus avec plus de $400 \mathrm{~Bq} \mathrm{~cm}^{-2}$. Cette valeur pourrait s'expliquer par une transpiration importante décrite par le patient et un lavage peu fréquent des mains du patient en question ( 1 à 2 fois pour ce patient, contre 3 à 8 fois pour les autres patients). L'activité surfacique au niveau du front (Fig. 5B) est dans la majorité des cas inférieure à $50 \mathrm{~Bq} \mathrm{~cm}^{-2}$ avec un maximum à $t=24 \mathrm{~h}$.

\subsection{Contamination des sols}

L'activité des sols en Figure 6 est en moyenne en dessous de $1 \mathrm{~Bq} \mathrm{~cm}^{-2}$.

\section{Discussion}

Sur la Figure 7, nos résultats sont comparés à deux autres publications : Ibis et al. (1992) et Hamizah et al. (2012).

\subsection{Contamination de la salive}

L'activité dans la salive est plus importante qu'attendue $\left(8,8 \mathrm{MBqg}^{-1}\right.$ en moyenne à $\left.t=4 \mathrm{~h}\right)$, mettant en évidence une activité jusqu'à 10 fois supérieure aux mesures publiées par Ibis et al. (1992). Une vigilance particulière doit donc être portée à tous les objets ayant été en contact avec la salive du patient. Durant l'hospitalisation, des masques chirurgicaux sont fournis aux patients qui ont pour consigne de les mettre

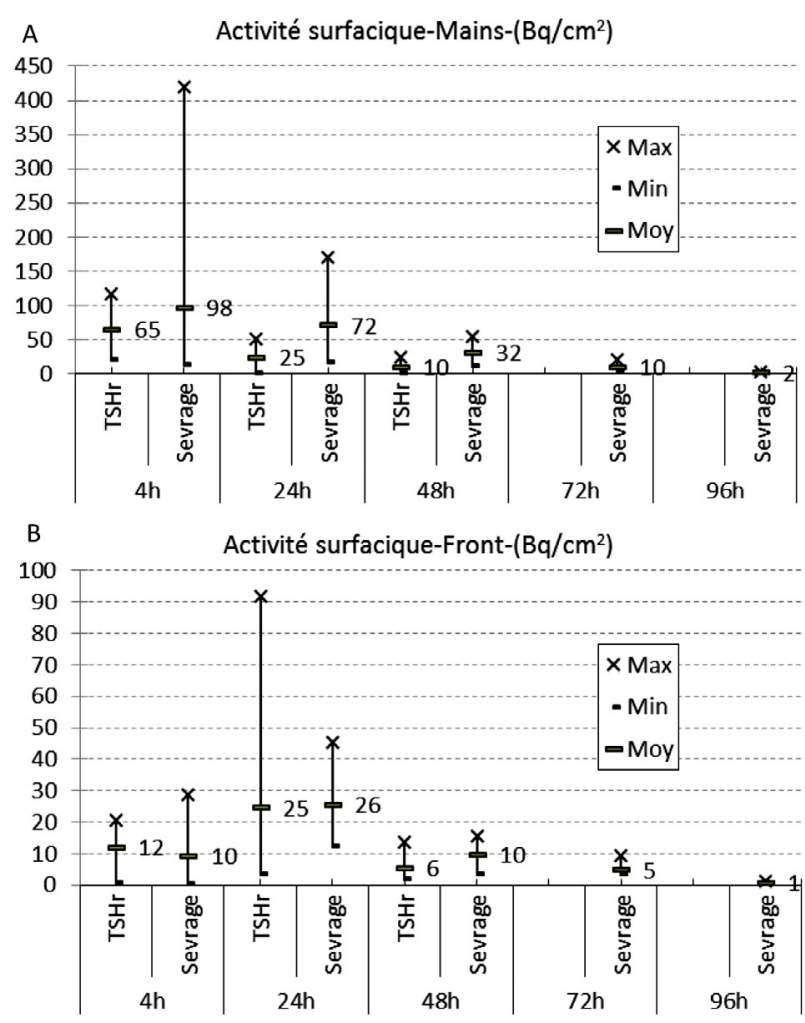

Figure 5. Activité surfacique de la peau au niveau des mains et du front.

Surface activity of the hands and forehead.

Activité sols $\left(\mathrm{Bq} / \mathrm{cm}^{2}\right)$

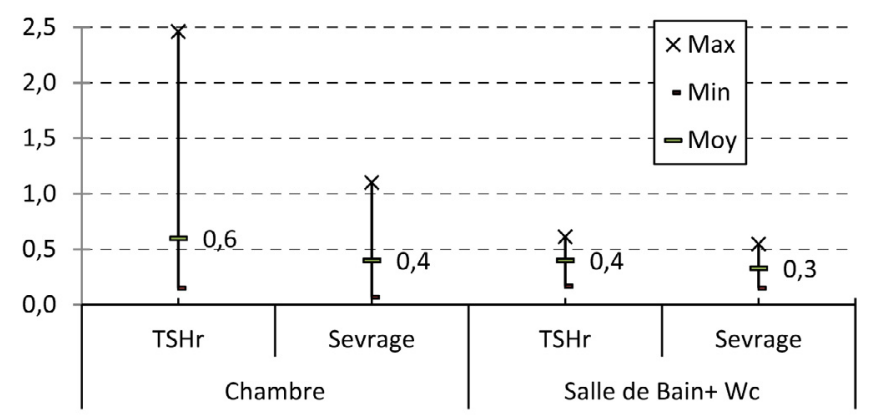

Figure 6. Contamination des sols.

Floor contamination.

lorsqu'un soignant entre dans la pièce. La radioactivité de ces masques a été quantifiée et des activités allant de 1 à $300 \mathrm{kBq}$ ont été mesurées par masque, montrant ainsi que ces masques constituent une barrière efficace à la dispersion d'iode-131 par la salive.

\subsection{Contamination cutanée}

Les activités surfaciques de la peau sont proches de Ibis et al. (1992) mais bien inférieures à Hamizah et al. (2012). Chez Ibis et al. (1992), la contamination de la peau est à son maximum à $24 \mathrm{~h}$ pour tous les frottis de la peau (front, nuque, poitrine, main). Dans notre étude, seules les mesures 

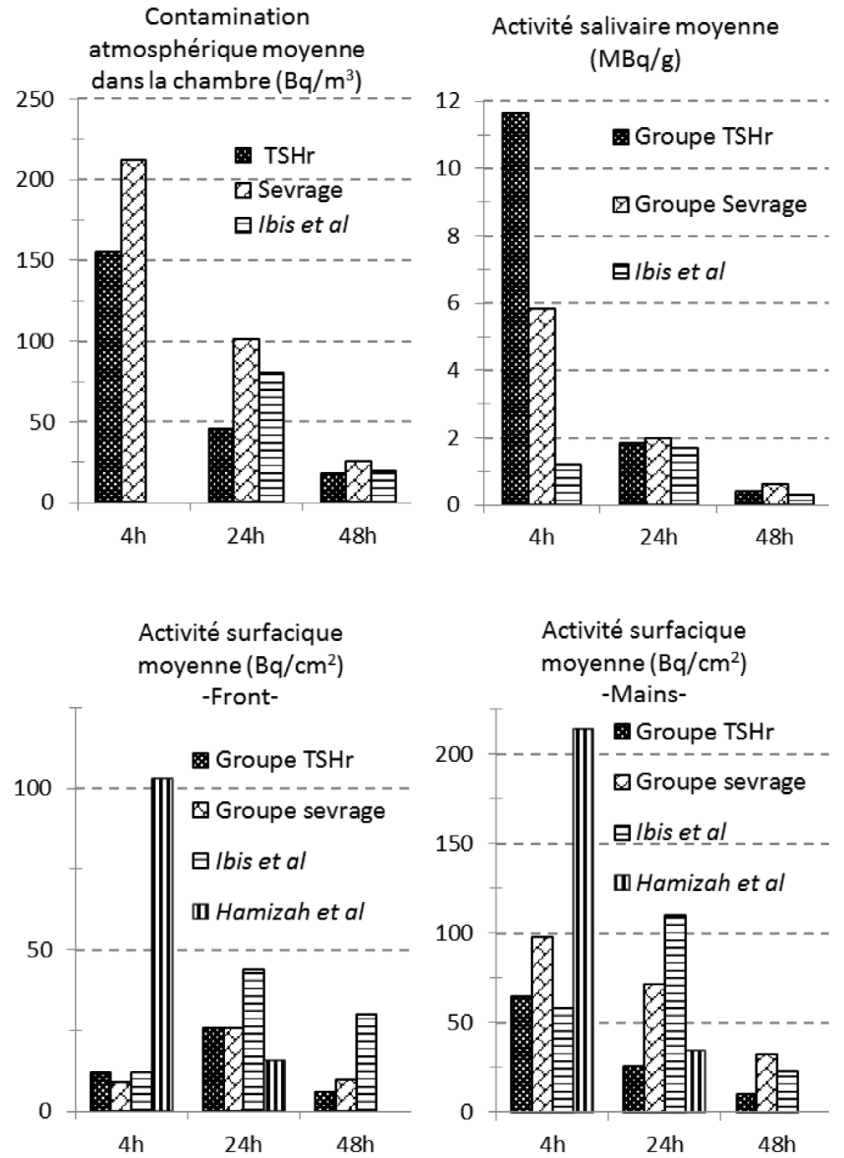

Figure 7. Comparaison avec d'autres études. Comparison with other publications.

du front des patients du groupe sevrage suivent cette tendance, les autres frottis présentant une diminution progressive de la contamination : les consignes de lavages fréquents des mains et de douches régulières, mises en place dans le service, semblent donc favoriser la diminution de l'activité surfacique de la peau.

\subsection{Contamination atmosphérique}

La contamination atmosphérique de la chambre peut atteindre plus de $700 \mathrm{~Bq} \mathrm{~m}^{-3}$. Ces niveaux de contamination de l'air sont en cohérence avec des mesures réalisées par l'Institut National de Recherche et de Sécurité (INRS, 2012).

Les concentrations atmosphériques sont proches dans les chambres et dans les WC. Il y a donc un équilibre qui se crée dans les deux pièces montrant l'absence de zone morte. La concentration particulièrement élevée dans les WC pour le patient No. 3 à $t=24 \mathrm{~h}$ s'explique par le fait que le patient a uriné pendant le prélèvement d'air. Cela montre qu'il y a une évaporation dans l'air quasi immédiate de l'iode-131 lors de la miction des patients et confirme la grande volatilité de l'iode131 déjà connue par un coefficient de volatilité élevé $(k=1)$ (Delacroix et al., 2004).
C'est chez le patient No. 5 dont la mesure de la contamination au niveau des mains était déjà la plus élevée (transpiration importante et lavages des mains moins fréquents) que la concentration atmosphérique dépasse les $700 \mathrm{~Bq} \mathrm{~m}^{-3}$. Le niveau de contamination atmosphérique semble donc être un témoin des différentes voies d'excrétion de l'iode-131 par les patients.

Les nouvelles règles d'aménagement des services de médecine nucléaire (Arrêté du 16 janvier 2015 portant homologation de la décision No. 2014-DC-0463 de l'Autorité de Sureté Nucléaire, 2015) qui imposent que les chambres RIV soient en dépression, sont justifiées au vue de ces résultats et apparaissent pertinentes pour limiter les risques de contamination des personnels via l'air de la chambre.

\subsection{Périodes effectives}

Les calculs de périodes effectives confirment une période effective légèrement plus courte pour le groupe TSHr (13,2 \pm $3,5 \mathrm{~h}$ contre 15,5 $\pm 2,3 \mathrm{~h}$ ) et sont en accord avec Remy et al. (2008) chez qui la différence entre les deux groupes est plus marquée $(10,5 \pm 1,5 \mathrm{~h}$ contre $15,2 \pm 4,7 \mathrm{~h})$ pour une cohorte de patient plus importante de 167 patients en sevrage et 36 sous TSHr. La petite taille de l'échantillon ne nous permet pas de conclure quant à la significativité de la différence.

\subsection{Impacts dosimétriques}

Comme attendu, les premières $24 \mathrm{~h}$ d'hospitalisation sont les heures où les risques d'exposition externe et de contamination du personnel sont les plus importants. En effet, le débit d'équivalent de dose ambiant à un mètre peut dépasser les $200 \mu \mathrm{Sv} \mathrm{h}^{-1}$ et la contamination atmosphérique de la chambre peut atteindre plus de $700 \mathrm{~Bq} \mathrm{~m}^{-3}$ conduisant à une dose engagée de $17 \mu \mathrm{Sv}$ en une heure pour un débit respiratoire de $1,2 \mathrm{~m}^{3} \mathrm{~h}^{-1}$ et une dose par unité d'incorporation de 2,0 $\times$ $10^{-8} \mathrm{~Sv} \mathrm{~Bq}^{-1}$ (ICRP 68, 1994). L'impact dosimétrique dû à l'inhalation d'iode-131 n'est donc pas négligeable mais à pondérer par rapport à l'exposition externe provenant du patient qui reste l'exposition la plus importante.

Les résultats montrent une très grande variabilité entre les patients. Cette variabilité provient probablement des différences individuelles physiologiques d'élimination de l'iode131 sur des patients pathologiques et, pour la contamination surfacique de la peau, de l'application inégale des consignes d'hygiène et de radioprotection par les patients (lavage des mains fréquent, douche quotidienne... ). En prenant en compte cette variabilité, et au vu des résultats qui montrent peu de différences entre les patients sous TSHr et en sevrage, il conviendra de mettre en place les mêmes recommandations de radioprotection pour ces deux modalités de prise en charge.

\section{Conclusion}

L'étude réalisée sur 12 patients a permis de mettre à jour les analyses de risque pour le personnel et de mettre en place 
des actions complémentaires de prévention de la contamination. Cette étude confirme des niveaux important d'iode-131 dans la salive de l'ordre du $\mathrm{MBqg}^{-1}$ de salive. Faire porter un masque au patient pendant la présence dans la chambre du personnel soignant est un moyen efficace pour protéger le personnel des projections de salive très concentrées en iode-131.

Concernant l'air de la chambre, les niveaux sont certes très variables d'un patient à l'autre, mais peuvent toutefois atteindre plus de $700 \mathrm{~Bq} \mathrm{~m}^{-3}$ dans les premières heures du traitement. Ces contaminations atmosphériques peuvent donc expliquer les contaminations internes du personnel ponctuellement détectées. Il conviendra donc de limiter au maximum le temps de présence du personnel soignant dans la chambre au moins dans les 24 premières heures. Réduire les passages dans la chambre en communiquant par téléphone avec le patient et limiter la surveillance (pouls, tension) sont des solutions pour restreindre le temps de présence dans la chambre.

Même si l'activité surfacique de la peau des patients reste faible (quelques dizaines de $\mathrm{Bq} \mathrm{cm}^{-2}$ ), on observe pour un patient de cette étude une augmentation significative de l'activité surfacique de la peau et de la contamination atmosphérique. La grande variabilité des mesures obtenues reflète la variabilité inter individuelle de l'excrétion par voie cutanée ou par exhalation. Pour la voie cutanée, les consignes au patient doivent donc être strictes sur le lavage fréquent des mains et du corps (douches quotidiennes) ce qui permettra d'éviter une éventuelle remise en suspension de l'iode-131 excrété par sudation.

Enfin, comme les niveaux de contamination et d'exposition sont quasi identiques pour les patients sous TSHr ou en sevrage, les mêmes recommandations doivent systématiquement être appliquées pour ces deux modalités de prise en charge.

\section{Annexe : Rendements de comptage}

\section{A.1 Méthodologie}

Pour les prélèvements dont l'activité est supérieure à $1 \mathrm{MBq}$ (limite inférieure de la plage d'utilisation respectant la linéarité), on réalise la mesure grâce à un activimètre MEDI 404 (Medisystem). L'incertitude relative sur l'activité mesurée est de $1,7 \%$ avec $k=1$ (CEA/LNHB-SFPM-SoFRa, 2006). Tous les autres prélèvements sont quantifiés grâce à un spectromètre gamma GC 1020 (Canberra) muni d'un détecteur au germanium et équipé du logiciel Génie 2000. Les comptages sont réalisés en prenant une fenêtre spectrométrique autour du pic de $364,5 \mathrm{keV}$ qui s'étend de 362,5 à $366,5 \mathrm{keV}$. Le temps d'acquisition dépend de l'activité des échantillons, il va de 60 à $1200 \mathrm{~s}$, l'objectif étant d'avoir une surface nette de pic d'au moins 1000 coups. Le logiciel calcule la surface nette $(N)$. L'incertitude sur le nombre d'impulsions nettes mesurées est estimée par $\sigma=\sqrt{ } N$.

Pour mesurer une activité en $\mathrm{Bq}$ de chaque prélèvement, il faut préalablement déterminer le rendement absolu d'absorption totale $(R \gamma)$ propre à chaque géométrie d'échantillon analysé.

$\gamma=\frac{N}{A \times I \gamma \times t}$, avec $N$ le nombre de coups dans la surface du pic ; $A$ l'activité en Becquerel ; I (81,2 \%) (Bé et al., 2008) l'intensité du gamma de $364,5 \mathrm{keV}$ et $t$ le temps en seconde.
Tableau A.2. Rendements absolus d'absorption totale des divers prélèvements.

Detector efficiency for different types of samples.

\begin{tabular}{ccc}
\hline Types d'échantillon & $\begin{array}{c}\text { Rendement absolu } \\
\text { d'absorption totale }\end{array}$ & $\begin{array}{c}\text { Incertitude relative (\%) } \\
\text { avec } \boldsymbol{k}=\mathbf{1}\end{array}$ \\
\hline Filtre charbon actif & $3,4 \times 10^{-2}$ & 2,5 \\
Salivette & $2,6 \times 10^{-3}$ & 1 \\
Frottis cutané & $3,5 \times 10^{-2}$ & 1,5 \\
Bandeau lavage sol & $1,5 \times 10^{-2}$ & 4 \\
Gaze pré-imprégnée & $2,5 \times 10^{-2}$ & 3,9 \\
\hline
\end{tabular}

Les rendements ont ainsi été déterminés pour chaque type de prélèvement à l'aide d'échantillons types pour lesquels les activités étaient connues. Des activités de l'ordre du MBq sont mesurées avec l'activimètre. Par dilution ou décroissance, des activités de l'ordre du $\mathrm{kBq}$ sont déposées sur les différents supports de prélèvements des échantillons dans la configuration de mesure utilisée. Pour les calculs d'incertitude des différents rendements, on néglige l'incertitude du temps de comptage et on prend une incertitude relative de $0,8 \%$ avec $k=1$ (Bé et al., 2008) sur l'intensité d'émission du gamma de $364,5 \mathrm{keV}$. Les incertitudes sur tous les rendements sont évaluées.

\section{A.2 Résultats}

Les rendements absolus d'absorption totale pour les différentes géométries d'échantillon sont présentés dans le Tableau A.2. Ils se situent entre 1,5 et 3,5\% excepté pour les prélèvements salivaires $(0,26 \%)$. Ce rendement plus faible pour les Salivette ${ }^{\circledR}$ s'explique par le fait que la radioactivité des échantillons de salives est beaucoup plus élevée que les autres échantillons. Afin d'éviter que le spectromètre gamma soit saturé, il faut placer le prélèvement plus loin du détecteur (à $8,5 \mathrm{~cm}$ au-dessus du cristal) ce qui diminue l'efficacité géométrique de la mesure.

\section{Références}

Arrêté du 16 janvier 2015 portant homologation de la décision No. 2014-DC-0463 de l'Autorité de sûreté nucléaire du 23 octobre 2014 relative aux règles techniques minimales de conception, d'exploitation et de maintenance auxquelles doivent répondre les installations de médecine nucléaire in vivo (2015).

Bé M.-M., Christé V., Dulieu C. (2008) NUCLÉIDE LARA Bibliothèque des émissions alpha, $\mathrm{X}$ et gamma. Rapport CEAR-6201.

CEA/LNHB-SFPM-SoFRa (2006) Guide d'utilisation et de contrôle qualité des activimètres.

Delacroix D., Guerre J.P., Leblanc P. (2004) Guide pratique Radionucléides et Radioprotection. EDP Sciences, Les Ulis. 
Hamizah N.M.Z., Juliana M.R., Waidi A.I., Ismalina S.N.I., Ahmad Z. (2012) Surface Contamination in Skin and Room during Hospitalization of Thyroid Cancer Patient Receiveing Radioiodine Ablation, JDMS 2, 27-33.

Ibis E., Wilson C.R., Collier B.D., Akansel G., Isitman A.T., Yoss R.G. (1992) Iodine-131 Contamination from Thyroid cancer patients, J. Nucl. Med. 33, 2110-2115.
ICRP Publication 68 (1994). Dose Coefficients for Intakes of Radionuclides by Workers, Ann. ICRP 24.

INRS FR8 (2012) Références en Santé et Travail. No. 131 :143-154.

Remy H., Borget I., Leboulleux S., Guilabert N., Lavielle F., Garsi J., Bournaud C., Gupta S., Schlumberger M., Ricard M. (2008) ${ }^{131}$ I Effective Half-Life and Dosimetry in Thyroid Cancer Patients, $J$. Nucl. Med. 49, 1445-1450.

Cite this article as: S. Guillot, P. Tylski, C. Scheiber, C. Bournaud-Salinas, C. Bolot, C. Harthe, P. Jalade. Quantification des risques de contamination et d'exposition externe du personnel en radiothérapie interne vectorisée par iode-131. Radioprotection 51(3), 171-177 (2016). 